\title{
An Efficient Response Distribution Function for 3D MIMO Channel Modeling from a Scatterer View
}

\author{
Sheng Deng ${ }^{1}$, Yuhao Wang ${ }^{1 *}$, \\ Jiangnan $\mathrm{Yu}^{1}$, Henry Leung ${ }^{2}$, Huilin Zhou ${ }^{1}$ and Bo Kong ${ }^{1}$ \\ ${ }^{1}$ School of Information Engineering, Nanchang University, China, 330031 \\ ${ }^{2}$ Department of Electrical and Computer Engineering, University of Calgary, \\ Calgary, ABT2N1N4, Canada. \\ E-mail:wangyuhao@ncu.edu.cn
}

\begin{abstract}
A Radio Environment Map (REM) maps its geolocation information to the propagation characteristics as complementary information for an adaptive communication of cognitive radio $(C R)$. In this paper we propose a novel theoretical three dimension (3D) Multiple Input Multiple Output (MIMO) channel model. The proposed model parameterizes the geolocation information of interacting obstacles referred as scatterers. An efficient response distribution function (ERDF) is developed to interpolate the array response for an arbitrary scatterer location. It is shown that the mutual information of the proposed model is consistent with a conventional geometry-based model with the lower simulation time. It allows us to generate channels with all possible scatterer distribution on the surface of a building effectively.
\end{abstract}

Keywords: channel model; Radio Environment Map (REM), MIMO, scatterer, Efficient Response Distribution Function (ERDF)

\section{Introduction}

A cognitive radio (CR) should be able to sense its surrounding environment and adapt to changing operating states in real-time [1-2]. As an integrated database for CRs, the radio environment map (REM) [3] has been widely studied recently. In order to support CR functionalities, the REM provides multi-domain environmental information and prior knowledge, such as geographical features, regulation, policy, radio equipment capability profile, and radio frequency (RF) emissions. Recent researches on REM pay much attention to cognitive radio network (CRN), whereas relative little works are done on the propagation channel among CR nodes [4-5]. Propagation channel characteristics are significant to make CRs an adaptive communication strategy. More information on the propagation channel characteristics are needed for the REM to better realize the CR capabilities. It is indispensable to develop an accurate and REM-friendly 3D MIMO channel model that have a more complete description of channel characteristics [6].

A comprehensive overview of the propagation models for CR design is given in [7]. It provides the key characteristics of wireless channel models to be used in the CR design. These key characteristics include path-loss, small-scale fading, delay spread, Doppler spread, angle spread, and they are hugely correlated [8, 9]. Conventional MIMO systems can be categorized two main types: physical models and analytical models [10]. We focus on the physical models because they are more accurate and have a wide spectrum for application. The geometry-based model is a kind of physical model that takes the geometry of a channel into account [11-12]. The double directional channel is a classical geometry-based model, where each path is described by parameters, such as the propagation delay, direction of arrival (DOA) and direction of departure (DOD) [13]. To develop the double directional channel model, a comprehensive model with a 
considerable number of parameters embracing the complete characterization in the time domain has been developed [14-16]. However, the models have to be refined if ultra wideband channels are used [17]. The equivalent frequency domain representation of the double directional channel model is given in [18]. In addition, the antenna response modeling is crucial in the geometry-based channel model, such as the angular domain representation, the effective aperture distribution function (EADF) [19], and the discrete vector spherical Fourier transform (DVSFT)-based model. The EADF is usually utilized to model a geometry-based channel due to its accuracy and low complexity (e.g. [20-22]).

However, the aforementioned MIMO channel models are not suitable for REM, as it requires evaluating $\mathrm{CR}$ performances under various propagation scenarios. In this paper REM processes the comprehensive information stored to produce a large amount of testing scenarios similar to CR environment, as a virtual "radio environment generator" [23]. There are two aspects required for conventional MIMO channel models to be ameliorated for REM. First, the information contained in REM depends on the geographic location. It is worthy to follow this feature in propagation modeling. REM graphical user interface (GUI) and communication devices are two potential types of REM users. Both of them can query the database of REM according to their geolocation in terms of latitude and longitude. Second, it is critical to merge faithful propagation models into REM without increasing the computational complexity because REM must supply current information in time. These are the two problems we try to address here to enhance the conventional MIMO channel models for REM.

Scatterers do not always disperse in the horizontal plane since they can be on the vertical plane when the transmitter $(\mathrm{Tx})$ and the receiver $(\mathrm{Rx})$ antenna arrays are located closely and are lower than the buildings [24]. Therefore, a 3D MIMO channel model is proposed here for REM. A comprehensive presentation of 3D channel modeling is given in [25]. A complete overview on the evolution of 3D channel modeling from theory to standardization can be found in [26] that illustrates a comparison among the frequently used models. A detailed overview on geometrically-based MIMO channel modeling with 3D channel models extension based on 2D channel models is also available in [11]. In this paper, we extend the double directional channel model in [13-16], where scatterers are disperse in the horizontal plane by using 3D spatial position of scatterers to describe MIMO channel characteristics in CR environment.

The contributions and novelties of this paper are summarized as follows:

1) We propose a novel theoretical 3D MIMO channel model from a scatterer view point, since the locations and coefficients of scatterers essentially determine the DOA, DOD and delay as well as the propagation channel characteristics. We choose the locations of scatterers and the scattering coefficients as parameters to describe a dynamic $3 \mathrm{D}$ radio environment. It has then be used to map the REM's geolocation information to the propagation characteristics. This will give us the capability to generate channels with all possible scatterers distribution on the surface of buildings.

2) We introduce an effective response distribution function (ERDF) to provide a continuous description of the relationship between scatterers and the channel impulse response. The ERDF can achieve high data compression since all antenna or array responses are concentrated in the transformed domain. We can then model the antenna or array response via an effective interpolation for arbitrary scatterer position. This information is used for the Cramer-Rao lower Bound (CRB) analysis of antenna array [27] and gradient-based parameter estimation techniques [18].

The remainder of this paper is organized as follows. Section II presents a brief review of the conventional geometry-based MIMO channel modeling approach. Section III discusses the basic geometric relationship between scatterers locations and the channel impulse responses and introduces the ERDF to improve representation of the relationship established. Section IV illustrates the reconstruction error of ERDF and compares the 
mutual information (MI) of the proposed model with that of the conventional radio channel model. Section V concludes the paper.

\section{A Brief Review of Conventional MIMO Channel Modeling}

A MIMO channel can be modeled either as double-directional channels or as radio channels between the location $\mathbf{r}_{T}$ of the Tx and the location $\mathbf{r}_{R}$ of the Rx. Regardless of polarization, impulse response of the double directional channel is a scalar sum of all individual multipath components (MPCs) independent of antenna number, type and configuration as well as system bandwidth. The delay spread, angle spread effects of time-variant channel is thus described by [13]

$$
h\left(\mathbf{r}_{T}, \mathbf{r}_{R}, \mathrm{t}, \tau, \Omega_{R}, \Omega_{T}\right)=\sum_{p=1}^{P} \alpha_{p} \delta\left(\tau-\tau_{p}\right) \delta\left(\Omega_{R}-\Omega_{R, p}\right) \delta\left(\Omega_{T}-\Omega_{T, p}\right)
$$

where $\alpha_{p}, \tau_{p}, \Omega_{R, p}$ and $\Omega_{T, p}$ denote the complex amplitude, delay, DOD and DOA of the $p$-th MPC, respectively, and ${ }^{p}$ is the number of MPCs. All parameters $\left(\mathbf{r}_{T}, \mathbf{r}_{R}, \alpha_{p}, \tau_{p}, \Omega_{R, p}, \Omega_{T, p}, P\right)$ in the above model may be functions of time for a dynamic propagation channel.

A MIMO channel consists of $M_{R} \times M_{T}$ channels of all received and transmitted antenna pairs, where $M_{R}$ and $M_{T}$ are the number of received and transmitted antennas, respectively. The radio channel incorporates the effects of antenna pattern and array configuration into the double directional impulse response given by [14-15]

$$
\mathbf{H}(t, \tau)=\sum_{p=1}^{P} \alpha_{p} \delta\left(\tau-\tau_{p}\right) \mathbf{a}_{R}\left(\Omega_{R, p}\right) \mathbf{a}_{T}^{H}\left(\Omega_{T, p}\right)
$$

where $\mathbf{a}_{R}\left(\Omega_{R, p}\right) \in \square^{M_{R} \times 1}$ and $\mathbf{a}_{T}\left(\Omega_{T, p}\right) \in \square^{M_{T} \times 1}$ represent the array response vectors on transmitted and received sides, respectively. It can be decomposed into a steering vector $\mathbf{a}_{\text {struct }}(\Omega)$ and a complex electric field pattern $b(\Omega)$ as

$$
\mathbf{a}\left(\Omega_{p}\right)=b\left(\Omega_{p}\right) \cdot \mathbf{a}_{\text {struct }}\left(\Omega_{p}\right)
$$

The steering vector $\mathbf{a}_{\text {struct }}\left(\Omega_{p}\right)$ is related to the antenna architectures. For example, the receive array response vectors for a uniform linear array (ULA), having element spacing $d_{r}$, is given by

$$
\mathbf{a}_{R, \mathrm{ULA}}\left(\Omega_{p}\right)=\left[1, e^{j 2 \pi d_{r} / \lambda \cos (\phi) \sin (\theta)}, \cdots, e^{j 2 \pi\left(M_{R}-1\right) d_{r} / \lambda \cos (\phi) \sin (\theta)}\right]
$$

where $\varphi$ and $\theta$ are the azimuth and elevation angles and $\Omega_{p}=(\varphi, \theta)$. The complex electric field pattern $b(\varphi, \theta)$ can be interpolated by EADF, sampled at arbitrary $\varphi$ and $\theta$, given by [19]

$$
b(\varphi, \theta)=\mathbf{d}_{b 1}^{H}(\theta) \cdot \mathbf{G} \cdot \mathbf{d}_{b 2}(\varphi)
$$

where $\mathbf{d}_{b 1}(\theta)=e^{j \theta \boldsymbol{\mu}_{1}} \in \square^{L_{1} \times 1}, \mathbf{d}_{b 2}(\varphi)=e^{j \varphi \boldsymbol{\mu}_{2}} \in \square^{L_{2} \times 1}$ and

$$
\begin{aligned}
& \boldsymbol{\mu}_{1}=\left[-\left(L_{1}-1\right) / 2,-\left(L_{1}-3\right) / 2, \cdots,\left(L_{1}-1\right) / 2\right]^{T} \in \square^{L_{1} \times 1}, \\
& \boldsymbol{\mu}_{2}=\left[-\left(L_{2}-1\right) / 2,-\left(L_{2}-3\right) / 2, \cdots,\left(L_{2}-1\right) / 2\right]^{T} \in \square^{L_{2} \times 1}
\end{aligned}
$$

The parameters $L_{1}$ and $L_{2}$ define the size of EADF matrix $G \in \square^{L_{1} \times L_{2}}$. 
In order to investigate the wideband channel, the equivalent frequency domain representation of (2) is given by [28]

$$
\mathbf{H}(t, f)=\sum_{p=1}^{P} \alpha_{p} e^{-j 2 \pi f \tau_{p}} \mathbf{a}_{R}\left(\Omega_{R, p}\right) \mathbf{a}_{T}^{H}\left(\Omega_{T, p}\right)
$$

Equation (6) is sampled in both time and frequency domains. The equal interval in the frequency domain $f_{0}$ and in the temporal domain $t_{0}$ sampling generates a set of

$$
\begin{aligned}
& M_{f} \times M_{t} \text { matrices } \mathbf{H}\left(m_{f} \cdot f_{0}, m_{t} \cdot t_{0}\right) \in \square^{M_{R} \times M_{T}} \text { where } m_{f}=-\left(M_{f}-1\right) / 2, \cdots+\left(M_{f}-1\right) / 2 \\
& \text { and } m_{t}=-\left(M_{t}-1\right) / 2, \cdots,+\left(M_{t}-1\right) / 2 \text {. Let } \\
& \qquad \mathbf{s}(t)=\left[\mathbf{s}\left(-\left(M_{f}-1 / 2\right) \cdot t_{0}\right), \mathbf{s}\left(m_{t} \cdot t_{0}\right), \cdots, \mathbf{s}\left(\left(M_{f}-1 / 2\right) \cdot t_{0}\right)\right] \in \square^{M_{f} M_{t} M_{R} M_{T} \times 1}
\end{aligned}
$$

where

$$
\mathbf{s}\left(m_{t} \cdot t_{0}\right)=\left[\mathbf{z}_{1,1}, \cdots, \mathbf{z}_{M_{R}, 1}, \mathbf{z}_{1, \mathrm{M}_{T}}, \cdots, \mathbf{z}_{M_{R}, \mathrm{M}_{T}}\right]^{T} \in \square^{M_{f} M_{R} M_{T} \times 1}
$$

and

$$
\mathbf{z}_{i j}=\left[\mathbf{H}\left(-\left(M_{f}-1 / 2\right) \cdot f_{0}\right)_{i, j}, \cdots, \mathbf{H}\left(+\left(M_{f}-1 / 2\right) \cdot f_{0}\right)_{i, j}\right]
$$

The vector $\mathbf{z}_{i j}$ contains the frequency domain samples for a channel between the ${ }^{j}$-th transmitted antenna and ${ }^{i}$-th received antenna. Each snapshot, a temporal domain sample can be seen as a time-invariant channel. The vector $\mathbf{z}_{i j}$ can be generalized by a modular expression with tensor products [18]

$$
\mathbf{s}(\boldsymbol{\theta}, t)=\mathbf{B}_{T} \diamond \mathbf{B}_{R} \diamond \mathbf{B}_{f} \cdot \boldsymbol{\alpha}
$$

where the elementary functions $\mathbf{B}_{f}, \mathbf{B}_{T}$ and $\mathbf{B}_{R}$ describe the effects of delay spread $(\tau)$, angle spread in Tx (DOD) and in Rx (DOA), respectively.

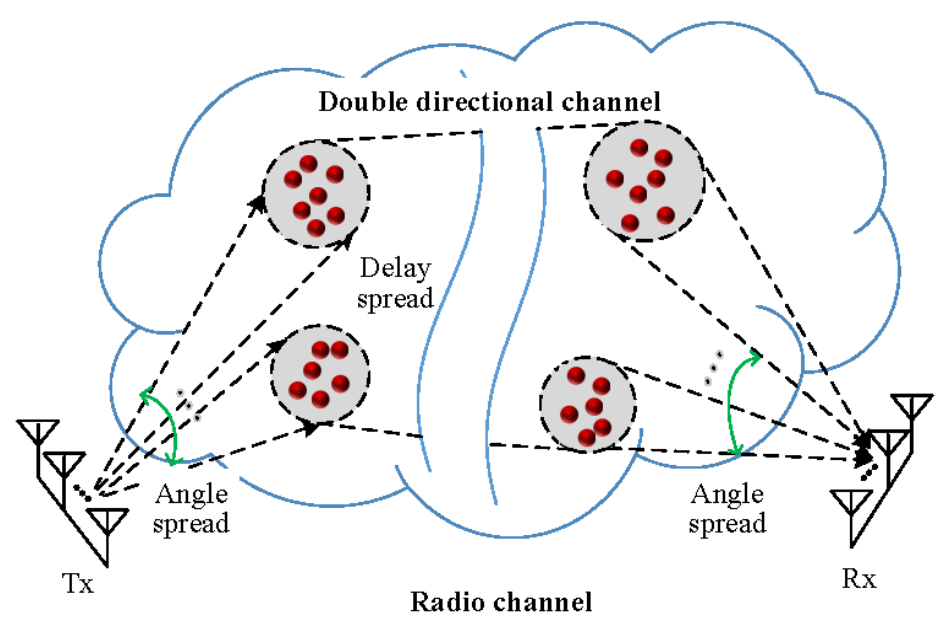

Figure 1. Principle of Conventional Radio Channel Model

Figure 1 shows the link between the double-directional model and radio channel in modeling principle. Both conventional models above describe the propagation processes from a terminal perspective, that is, the ends of path catch more attention instead of propagation geometry environment. Although these models describe the ends section of propagation well, they cannot reflect the geolocation information of the specific environments affects the propagation directly. 


\section{Proposed 3D MIMO Channel Model from A Scatterer View}

The propagation of electromagnetic (EM) waves is modeled as ray-like paths which link the Tx and Rx. The locality where the path interacts with a physical obstacle, such as a reflection, a scattering or a diffraction, are marked by a point-like interacting obstacle (IO) referred as scatterers [28]. Let us consider a wideband MIMO system with scatterers placed on buildings surface in a 3D space as illustrated in Figure 2(a). The location of scatterers determines the delay, azimuth and elevation of MPCs, and the geometric relationship among them can be established. In older to get a consistent way to describe the environment, the line of sight (LOS) propagation is also seen as a scatterer at the middle of the path. Here, we work on mapping the geolocation information of scatterers to channel impulse response.

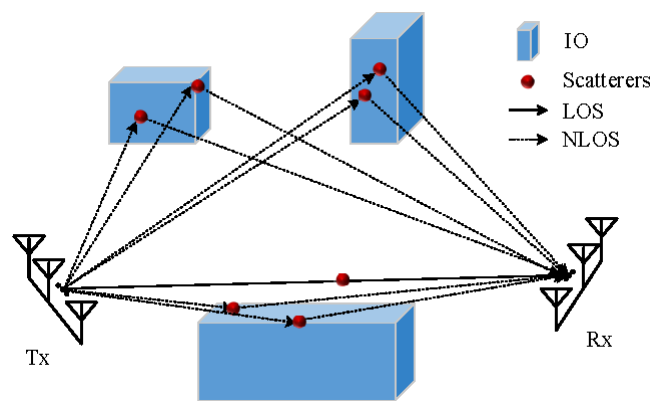

(a)

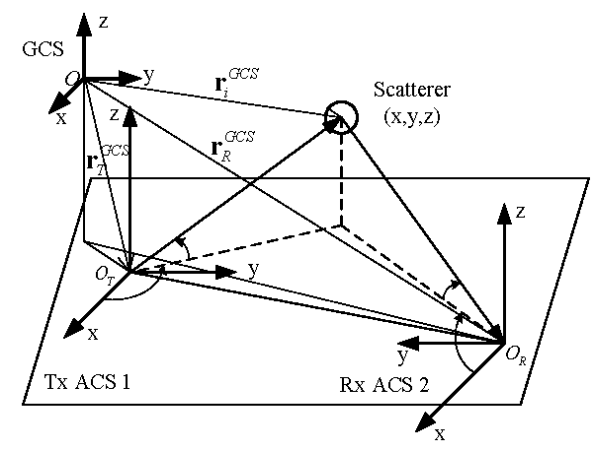

(b)

Figure 2. Basic Geometric Description of (a) a 3D MIMO Channel with Scatterers on the Surface of the Buildings (b) a Specific Single sCatterer (circle) with Three Cartesian Coordinate Systems (GCS, ACS1, ACS2)

\subsection{General Description with Scatterers' Locations}

To understand the MIMO system as well as the computations required, three Cartesian coordinate systems are employed as shown in Figure 2(b) The global coordinate system (GCS) allows all scatterers, obstacles, and antenna arrays positioning. The two array coordinate systems (ACS1 and ACS2) are used to describe the positions of single elements constituting the antenna array of transmitter and receiver respectively. A singlescatterer is set in the arbitrary 3D space as an example, and the directions of propagation rays are represented by the arrowed lines.

In GCS, the coordinates of locations of all $I$ scatterers are represented in the collection $\chi^{G C S}=\left\{\left(x_{i}^{G C S}, \mathrm{y}_{i}^{G C S}, \mathrm{z}_{i}^{G C S}\right) \mid i=1, \ldots, \mathrm{I}\right\}$ $\mathbf{r}_{i}^{G C S}=\left[x_{i}^{G C S}, y_{i}^{G C S}, z_{i}^{G C S}\right]^{T}$. The location of the $\mathrm{Tx}$ and $\mathrm{Rx}$ array is defined as $\mathbf{r}_{T}^{G C S}=\left[x_{T}^{G C S}, y_{T}^{G C S}, z_{T}^{G C S}\right]^{T}$ and $\mathbf{r}_{R}^{G C S}=\left[x_{R}^{G C S}, y_{R}^{G C S}, z_{R}^{G C S}\right]^{T}$ respectively. The initial positions of the center of transmitter array: $O_{T}\left(a_{T}^{G C S}, b_{T}^{G C S}, c_{T}^{G C S}\right)$ and receiver array: $O_{R}\left(a_{R}^{G C S}, b_{R}^{G C S}, c_{R}^{G C S}\right)$ are assumed to be constant and known.

The signal over a single path with an electrical length of $l_{i}$ at the wideband result in different phase shifts among the frequency points. To express the frequency dependence of phase shifts, we define a vector-valued function 


$$
\mathbf{a}_{l}\left(\mathbf{r}_{i}^{G C S}\right)=e^{-j 2 \pi\left(\mathbf{f}+f_{c}\right) \cdot l_{i}\left(\mathbf{r}_{i}^{G C S}\right) / c}
$$

where the distance ${ }^{l_{i}\left(\mathbf{r}_{i}^{G C S}\right)=\left\|\mathbf{r}_{i}^{G C S}-\mathbf{r}_{T}^{G C S}\right\|+\left\|\mathbf{r}_{i}^{G C S}-\mathbf{r}_{R}^{G C S}\right\|}$ is defined with respect to the centers of arrays, and the vector $\mathbf{f}=\left[-\left(M_{f}-1\right) / 2, \cdots,\left(M_{f}-1\right) / 2\right]^{T} \cdot f_{0}$ is the sampling frequency bins. The operator $\|\cdot\|$ denotes the Euclidean norm, and $c$ is the speed of light. $\mathbf{a}_{p}\left(\mathbf{r}_{i}^{G C S}\right)$ is sampled with the equidistant sampling interval $f_{0}$ at $M_{f}$ frequency points.

Since our model considers the effects of antenna array, it is crucial to describe array response vectors for the common antenna array configurations in the ACS, such as the uniform linear array (ULA) and the uniform circular array (UCA). Here we use ULA as an example and the results can be extended to other types of array.

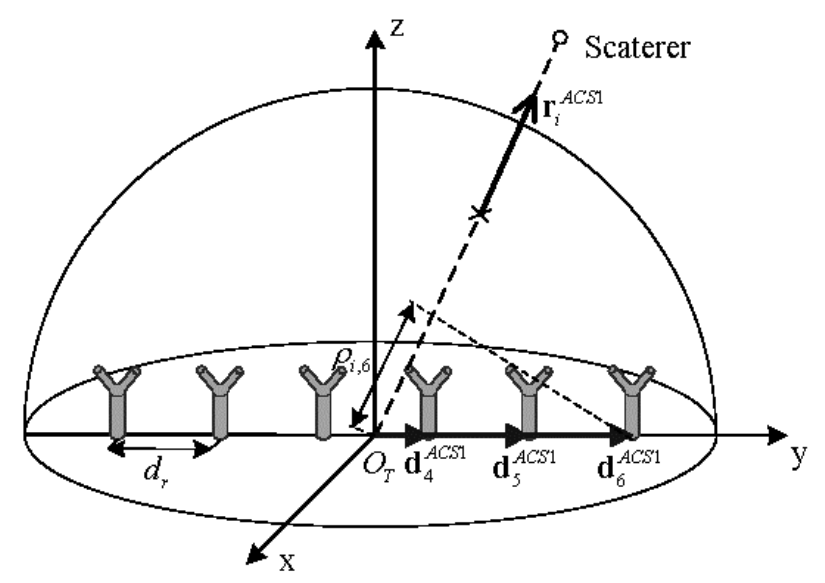

Figure 3. The Coordinate System for a ULA in Tx (ACS1) and an Impinging Planar Wavefront

The structure of the antenna array is described in the ACS as shown in Figure 3. Let $\chi^{A C S 1}=\left\{x_{i}^{A C S 1}, y_{i}^{A C S 1}, z_{i}^{A C S 1} \mid i=1, \ldots, \mathrm{I}\right\}$ the vector $\mathbf{r}_{i}^{A C S 1}=\left[x_{i}^{A C S 1}, y_{i}^{A C S 1}, z_{i}^{A C S 1}\right]^{T}$ stands for the direction from the center of array to the ${ }^{i}$-th scatterer. The position vector of $m_{\text {-th antenna in ACS1 becomes }}$

$$
\mathbf{d}_{m}^{A C S 1}=\left[0, \frac{-M_{R}+2 m-1}{2} \cdot d_{r}, 0\right], m=1, \ldots, \mathrm{M}_{R}
$$

where $M_{R}$ denotes the number of antennas in $\mathrm{Rx}$, and $d_{r}$ is the antenna spacing. The wave path difference of antennas in reference to the center of array is computed as

$$
\rho_{i, m}=\mathbf{d}_{m}^{A C S 1} \cdot \tilde{\mathbf{r}}_{i}^{A C S 1}
$$

where $\mathbf{r}_{i}^{A C S 1}=\mathbf{r}_{i}^{A C S 1} /\left\|\mathbf{r}_{i}^{A C S 1}\right\|$ is the normalization direction vector.

The array response vectors $\mathbf{a}\left(\mathbf{r}_{i}^{A C S 1}\right)$ can then be decomposed as follows

$$
\mathbf{a}\left(\mathbf{r}_{i}^{A C S 1}\right)=\mathbf{a}_{U L A}\left(\mathbf{r}_{i}^{A C S 1}\right) \cdot b\left(\mathbf{r}_{i}^{A C S 1}\right)
$$

where the steering vector is defined by

$$
\mathbf{a}_{U L A}\left(\mathbf{r}_{i}^{A C S 1}\right)=\left[e^{-j 2 \pi \rho_{i, 1} / \lambda}, e^{-j 2 \pi \rho_{i, \mathrm{~m}} / \lambda}, \cdots, e^{-j 2 \pi \rho_{i, M_{R}} / \lambda}\right]^{T} \in \square^{M_{R} \times 1}
$$


and antenna beam pattern $b\left(\mathbf{r}_{i}^{A C S 1}\right)$ is a scalar. The array response vectors $\mathbf{a}\left(\mathbf{r}_{i}^{A C S 2}\right)$ of the receiver array can also be described in the same way. Note that the DOA toward the array is the opposite direction of departure ${ }^{A C S 1}$ given by

$$
\mathbf{r}_{i}^{A C S 2}=-\left[x_{i}^{A C S 2}, y_{i}^{A C S 2}, z_{i}^{A C S 2}\right]^{T}
$$

Since the coordinates of scatterers in GCS are chosen as the parameters of the proposed model and the coordinates parameters of the proposed model are not suitable for the traditionally beam pattern, we need to perform the transformation $\mathbf{r}^{G C S} \rightarrow \mathbf{r}^{A C S} \rightarrow \boldsymbol{\Omega}=(\varphi, \theta)$. The homogeneous coordinates [28] is employed here to perform the transformation from GCS to ACS.

In order to define the position of one coordinate system inside another, there are at least six independent parameters to describe the orientation and translation of an orthonormal basis in 3D space, namely, three rotation angles: $\omega_{x}, \omega_{y}, \omega_{z}$ and three translation coordinates: $x, y, z$. For instance, Figure 2(b) illustrates the transformation of the GCS to the ACS: the translation can be calculated as the addition of the translation vector $\mathbf{T}^{G C S}=\left[x^{G C S}, \mathrm{y}^{G C S}, z^{G C S}\right]^{T}$; the orientation of ACS is rotated for the three rotation angles $\omega_{x}^{G C S}, \omega_{y}^{G C S}$ and $\omega_{z}^{G C S}$ around the $\mathrm{x}-, \mathrm{y}$ - and $\mathrm{z}$-axis in respect to the GCS respectively. Let $\mathbf{r}^{G C S}=\left[x^{G C S}, \mathrm{y}^{G C S}, z^{G C S}\right]^{T}$ be a point to be transformed and $\mathbf{r}^{A C S}=\left[x^{A C S}, \mathrm{y}^{A C S}, z^{A C S}\right]^{T}$

system. Correspondingly, $\quad \mathbf{r}_{\text {hom }}^{G C S}=\left[x^{G C S}, \mathrm{y}^{G C S}, z^{G C S}, 1\right]^{T}$ is the original point and $\mathbf{r}_{\text {hom }}^{A C S}=\left[x^{A C S}, \mathrm{y}^{A C S}, z^{A C S}, 1\right]^{T}$ is the transformed point in homogeneous coordinates system. The transformations can be expressed as

$$
\mathbf{r}_{\text {hom }}^{A C S}=\mathbf{Q} \cdot \mathbf{r}_{\text {hom }}^{G C S}
$$

where

$$
\mathbf{Q}=\operatorname{Trans}\left(\mathbf{T}^{G C S}\right) \cdot \operatorname{Rot}_{z}\left(\omega_{z}^{G C S}\right) \cdot \operatorname{Rot}_{y}\left(\omega_{y}^{G C S}\right) \cdot \operatorname{Rot}_{x}\left(\omega_{x}^{G C S}\right)
$$

and

$$
\begin{gathered}
\operatorname{Trans}\left(\mathbf{T}^{G C S}\right)=\left[\begin{array}{cccc}
1 & 0 & 0 & x^{G C S} \\
0 & 1 & 0 & y^{G C S} \\
0 & 0 & 1 & z^{G C S} \\
0 & 0 & 0 & 1
\end{array}\right] \\
\operatorname{Rot}_{x}\left(\omega_{x}^{G C S}\right)=\left[\begin{array}{cccc}
1 & 0 & 0 & 0 \\
0 & \cos \left(\omega_{x}^{G C S}\right) & -\sin \left(\omega_{x}^{G C S}\right) & 0 \\
0 & \sin \left(\omega_{x}^{G C S}\right) & \cos \left(\omega_{x}^{G C S}\right) & 0 \\
0 & 0 & 0 & 1
\end{array}\right]
\end{gathered}
$$




$$
\begin{aligned}
\operatorname{Rot}_{y}\left(\omega_{y}^{G C S}\right) & =\left[\begin{array}{cccc}
\cos \left(\omega_{y}^{G C S}\right) & 0 & \sin \left(\omega_{y}^{G C S}\right) & 0 \\
0 & 1 & 0 & 0 \\
-\sin \left(\omega_{y}^{G C S}\right) & 0 & \cos \left(\omega_{y}^{G C S}\right) & 0 \\
0 & 0 & 0 & 1
\end{array}\right] \\
\operatorname{Rot}_{z}\left(\omega_{z}^{G C S}\right) & =\left[\begin{array}{cccc}
\cos \left(\omega_{z}^{G C S}\right) & -\sin \left(\omega_{z}^{G C S}\right) & 0 & 0 \\
\sin \left(\omega_{z}^{G C S}\right) & \cos \left(\omega_{z}^{G C S}\right) & 0 & 0 \\
0 & 0 & 1 & 0 \\
0 & 0 & 0 & 1
\end{array}\right]
\end{aligned}
$$

Hence, the homogeneous coordinates combine all the transformations of three rotation matrixes: $\operatorname{Rot}_{x}\left(\omega_{x}^{G C S}\right), \operatorname{Rot}_{y}\left(\omega_{y}^{G C S}\right)$ and $\operatorname{Rot}_{z}\left(\omega_{z}^{G C S}\right)$ as well as a translation matrix $\operatorname{Trans}\left(\mathbf{T}^{G C S}\right)$ into one 4-by-4 matrix $\mathbf{Q}$.

The coordinates of scatterers in GCS ( i.e. $\chi^{G C S}$ ) are chosen as the input parameters, and the corresponding coordinates in ACS1 and ACS2 can be transformed with the help of homogeneous coordinate, that is

$$
\mathbf{r}_{\text {hom, }}^{A C S 1}=\mathbf{Q}_{G C S}^{A C S 1} \cdot \mathbf{r}_{\text {hom, } \mathrm{i}}^{G C S}
$$

and

$$
\mathbf{r}_{\text {hom, }}^{A C S 2}=\mathbf{Q}_{G C S}^{A C S 2} \cdot \mathbf{r}_{\text {hom, }}^{G C S}
$$

where $\mathbf{Q}_{G C S}^{A C S 1}$ and $\mathbf{Q}_{G C S}^{A C S 2}$ denote the transformations of GCS to ACS1 and ACS2, respectively. ${ }_{i}^{A C S 1}$ and $\mathbf{r}_{i}^{A C S 2}$ can then be obtained from the front three coordinates of $\mathbf{r}_{\text {hom, } \mathrm{i} \text { and }}^{A C S 1} \mathbf{r}_{\mathrm{hom}, \mathrm{i}}^{A C S 2}$.

The antenna beam pattern can now be described with the locations of scatterers. The beam pattern is stored as a matrix with respect to the azimuth and elevation. In order to utilize the data of the beam pattern matrix, the azimuth ${ }^{\varphi}$ and elevation $\theta$ of each scatterers $\mathbf{r}_{\left(k_{x}, k_{y}, k_{z}\right)}^{G C S}$ need to be calculated as

$$
\left\{\begin{array}{l}
\mathbf{r}_{\text {hom, }}^{A C S}=\mathbf{Q}_{G C S}^{A C S} \cdot \mathbf{r}_{\text {hom, }}^{G C S} \\
\varphi=\operatorname{atan} 2\left(y^{A C S}, x^{A C S}\right) \\
\theta=\operatorname{atan} 2\left(z^{A C S}, \sqrt{\left(y^{A C S}\right)^{2}+\left(x^{A C S}\right)^{2}}\right)
\end{array}\right.
$$

where the superscript ACS can be substituted for ACS1 and ACS2 to calculate the DOD and DOA respectively.

Using the EADF, the $b\left(\mathbf{r}^{G C S}\right)$ can be obtained by putting the calculated azimuth $\varphi$ and elevation $\theta$ into (5). For $I$ single-scatterers in the propagation environment, the wideband MIMO radio channel model can be expressed in the following form

$$
\left\{\begin{array}{l}
\mathbf{s}(\chi)=\sum_{i=1}^{I} \gamma_{i} \cdot \mathbf{a}_{T_{X}}\left(\mathbf{r}_{i}^{A C S 1}\right) \otimes \mathbf{a}_{R x}\left(\mathbf{r}_{i}^{A C S 2}\right) \otimes \mathbf{a}_{l}\left(\mathbf{r}_{i}^{G C S}\right) \\
\mathbf{r}_{\text {hom, }}^{A C S 1}=\mathbf{Q}_{G C S}^{A C S 1} \cdot \mathbf{r}_{\text {hom, }}^{G C S} \\
\mathbf{r}_{\text {hom }, \mathrm{i}}^{A C S 2}=\mathbf{Q}_{G C S}^{A C S 2} \cdot \mathbf{r}_{\text {hom, },}^{G C S}
\end{array}\right.
$$


where the extended collection $\chi=\left\{\left(x_{i}^{G C S}, \mathrm{y}_{i}^{G C S}, \mathrm{z}_{i}^{G C S}, \gamma_{i}\right) \mid i=1, \ldots, I\right\}$ contains all the parameters of the proposed model. The complex scattering coefficient $\gamma_{i}$ describes the effects such as loss on the interaction points, which can be considered as frequency independent.

\subsection{The Proposed Model with Effective Response Distribution Function}

The transformations in (26) lead to a model with a high computational complexity and it is difficult to analyze its derivatives with respect to the parameters for applications, such as gradient based parameter estimation techniques. To address this problem, we introduce an efficient representation of the array response with respect to the 3D Cartesian coordinates, which we refer to as the Effective Response Distribution Function (ERDF). The ERDF provides an efficient interpolation method so that the model could have a continuous description of the antenna array response. According to (26), the model can be rewritten as

$$
\mathbf{s}(\chi)=\sum_{i=1}^{I} \gamma_{i} \cdot \underbrace{\left\{\mathbf{a}_{T x}^{U L A}\left(\mathbf{r}_{i}^{A C S 1}\right) \otimes \mathbf{a}_{R x}^{U L A}\left(\mathbf{r}_{i}^{A C S 2}\right)\right\} \cdot \underbrace{\left\{b_{T x}\left(\mathbf{r}_{i}^{A C S 1}\right) \cdot b_{R x}\left(\mathbf{r}_{i}^{A C S 2}\right)\right\}}_{\mathrm{I}}}_{\mathrm{II}} \otimes \mathbf{a}_{l}\left(\mathbf{r}_{i}^{G C S}\right)
$$

where part I refers to the antenna response (ANR) defined by

$$
b_{\text {ant }}\left(\mathbf{r}_{i}^{G C S}\right)=b_{\text {ant }}\left(\mathbf{r}_{i}^{A C S 1}, \mathbf{r}_{i}^{A C S 2}\right)=b_{T x}\left(\mathbf{r}_{i}^{A C S 1}\right) \cdot b_{R x}\left(\mathbf{r}_{i}^{A C S 2}\right)
$$

and part II refers to the antenna array response (AAR) defined by

$$
\mathbf{b}_{\text {array }}\left(\mathbf{r}_{i}^{G C S}\right)=\mathbf{b}_{\text {array }}\left(\mathbf{r}_{i}^{A C S 1}, \mathbf{r}_{i}^{A C S 2}\right)=\mathbf{a}_{T x}^{U L A}\left(\mathbf{r}_{i}^{A C S 1}\right) \otimes \mathbf{a}_{R x}^{U L A}\left(\mathbf{r}_{i}^{A C S 2}\right) b_{T x}\left(\mathbf{r}_{i}^{A C S 1}\right) b_{R x}\left(\mathbf{r}_{i}^{A C S 2}\right)
$$

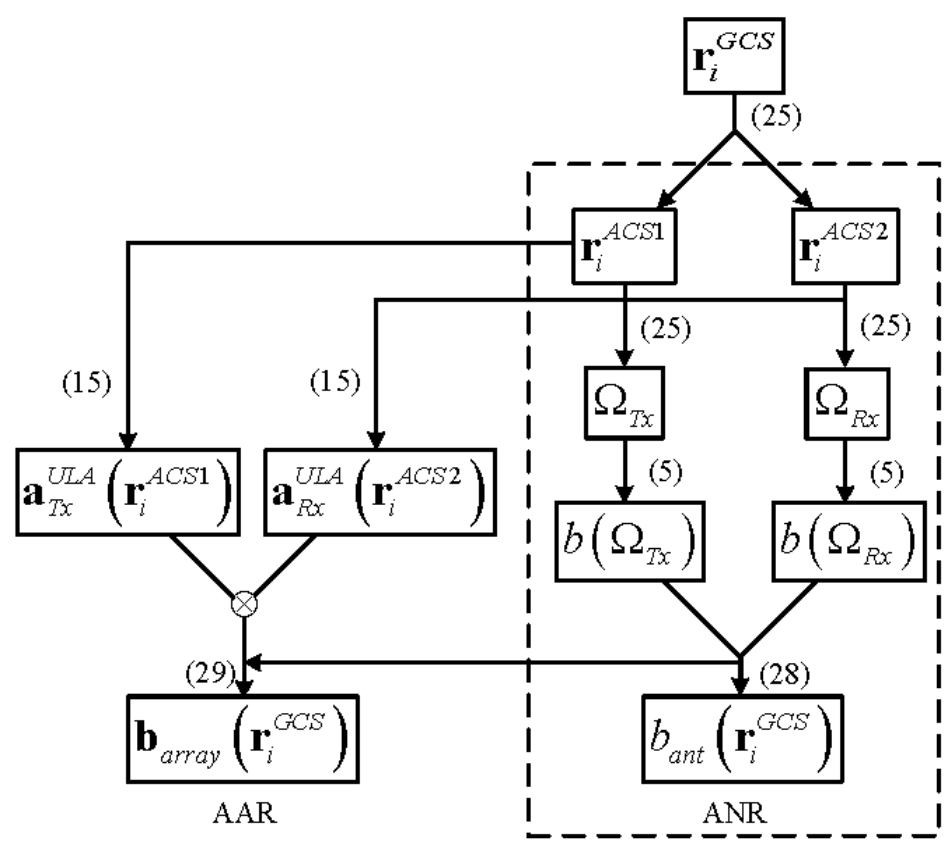

Figure 4. Diagram of the AAR and ANR Calculation

Figure 4 shows the detailed procedure for the AAR and ANR calculations. The data shown in the box and the processes are represented with arrows marked by the equation number. Note that the scalar ANR $b_{a n t}\left(\mathbf{r}_{i}^{G C S}\right)$ ignores the effects of the antenna architectures whereas the vector AAR 


$$
\mathbf{b}_{\text {array }}\left(\mathbf{r}_{i}^{G C S}\right)=\left[\begin{array}{lll}
b_{1,1}\left(\mathbf{r}_{i}^{G C S}\right) & \cdots & b_{\mathrm{M}_{T}, \mathrm{M}_{R}}\left(\mathbf{r}_{i}^{G C S}\right)
\end{array}\right]^{T}
$$

collects the different ANRs between the MIMO channels of all received and transmitted antenna pairs.

Both ANR and AAR are represented by the ERDF to resolve the issues of (26). The idea of ERDF is to compress the data of the responses at all 3D sampling grid as preprocessing and then to model the responses as an interpolation. This approach is basically similar to the EADF [19]. The difference is that the EADF is an alternative expression of antenna beam pattern for an arbitrary direction in azimuth and elevation, whereas the ERDF is an efficient way to calculate the response for an arbitrary scattering position in 3D Cartesian coordinates. In other words, ERDF directly maps continuous location $\mathbf{r}^{G C S}$ to the ANR or AAR.

We propose two models based on the ERDF of AAR and ANR respectively. Let GCS represent the $3 \mathrm{D}$ space with the origin of coordinates centering the space. It range from $-l_{x}$ to $+l_{x}$ along the $\mathrm{x}$-axis, ${ }^{-l_{y}}$ to ${ }^{+l_{y}}$ along the y-axis, and from $-l_{z}$ to $+l_{z}$ along the $\mathrm{z}-$ axis. Besides the chosen cube range, ERDF can be applied in arbitrary ranges of scenarios. Let $\delta_{x}, \delta_{y}$ and $\delta_{z}$ be the sampling interval for the coordinates of $x, y$ and $z$, respectively. There are $M_{x}, M_{y}$ and $M_{z}$ sampling numbers in the three axes and $M_{3 D}$ sampling points in the grid of 3D space, where $M_{3 D}=M_{x} \cdot M_{y} \cdot M_{z}$. Let $\mathbf{r}_{\left(k_{x}, k_{y}, k_{z}\right)}^{G C S}$ denote the $3 \mathrm{D}$ sampling points, where $k_{x}=-\left(M_{x}-1\right) / 2, \ldots,\left(M_{x}-1\right) / 2$, $k_{y}=-\left(M_{y}-1\right) / 2, \ldots,\left(M_{y}-1\right) / 2$ and $k_{z}=-\left(M_{z}-1\right) / 2, \ldots,\left(M_{z}-1\right) / 2$.

3.2.1. Model I: The spatial domain sampling of (28) at $M_{3 D}$ points yield a set of $M_{3 D}$ ANRs $b_{\text {ant }}\left(\mathbf{r}_{\left(k_{x}, k_{y}, k_{z}\right)}^{G C S}\right)$. Collecting the computed complex values $b_{\text {ant }}\left(\mathbf{r}_{\left(k_{x}, k_{y}, k_{z}\right)}^{G C S}\right)$, the ANRs distribution can be expressed in the tensor form as $\mathbf{B}_{\text {ant }} \in \square^{M_{x} \times M_{y} \times M_{z}}$, where $\mathbf{B}_{\text {ant }}\left(k_{x}, k_{y}, k_{z}\right)=b_{a n t}\left(\mathbf{r}_{\left(k_{x}, k_{y}, k_{z}\right)}^{G C S}\right)$.The tensor $\mathbf{B}_{\text {ant }}$ can be transformed via a three dimensional Discrete Fourier Transform (3D DFT). The resulting function $\mathbf{R}_{\text {ant }} \in \square^{M_{x} \times M_{y} \times M_{z}}$ represents the ERDF of ANR. The elements of the transformed tensor $\mathbf{R}_{\text {ant }}$ can be obtained by

$$
\mathbf{R}_{a n t}(u, v, w)=\operatorname{vec}\left(B_{a n t}\right)^{T} \cdot\left(\mathbf{e}_{z}(u) \otimes \mathbf{e}_{y}(v) \otimes \mathbf{e}_{x}(w)\right)
$$

where $\mathbf{e}_{x}(u)=e^{-j u \cdot \mathbf{X}}, \mathbf{e}_{y}(v)=e^{-j v \cdot \mathbf{Y}}, \mathbf{e}_{z}(w)=e^{-j w \cdot \mathbf{Z}}$

$$
\begin{aligned}
& \mathbf{X}=\frac{2 \pi}{M_{x} \delta_{x}} \cdot\left[-l_{x}, \cdots,-l_{x}+m \delta_{x}, \cdots,+l_{x}\right]^{T}, m=0, \ldots, \mathbf{M}_{x}-1 \\
& \mathbf{Y}=\frac{2 \pi}{M_{y} \delta_{y}} \cdot\left[-l_{y}, \cdots,-l_{y}+m \delta_{y}, \cdots,+l_{y}\right]^{T}, m=0, \ldots, \mathbf{M}_{y}-1 \\
& \mathbf{Z}=\frac{2 \pi}{M_{z} \delta_{z}} \cdot\left[-l_{z}, \cdots,-l_{z},+m \delta_{z}, \cdots,+l_{x}\right]^{T}, m=0, \ldots, \mathbf{M}_{z}-1
\end{aligned}
$$

and $u=-\left(M_{x}-1\right) / 2, \ldots,\left(M_{x}-1\right) / 2, v=-\left(M_{y}-1\right) / 2, \ldots,\left(M_{y}-1\right) / 2$, $v=-\left(M_{z}-1\right) / 2, \ldots,\left(M_{z}-1\right) / 2$ denotes the sampling points of transformed space. 


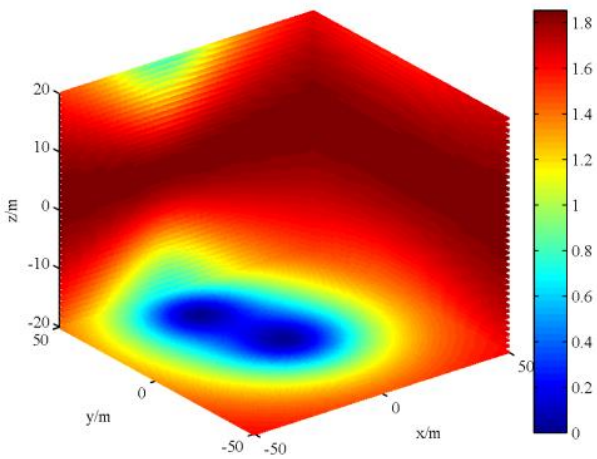

(a)

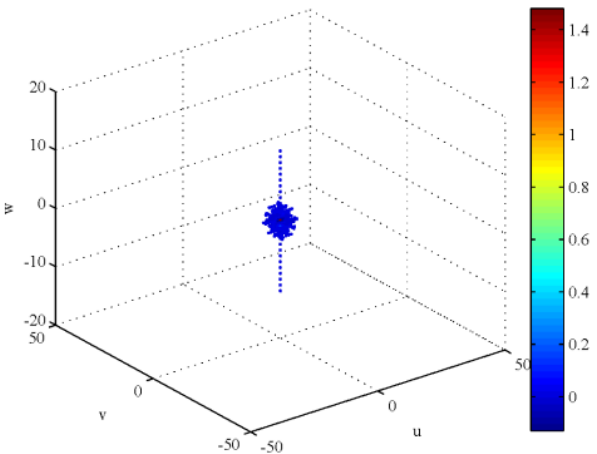

(b)

Figure 5. Representations of (a) the Absolute Values of the ANR at the Spatial Sampling Points and (b) the Corresponding ERDF Containing Approximately 300 values

Figure 5(a) shows the ANR tensor $\mathbf{B}_{\text {ant }}$ of a $100 \times 100 \times 40$ space. Figure 5(b) displays the absolute values of the corresponding transformed tensor $\mathbf{R}_{a n t}$. The equivalent ANRERDF only needs approximately $M_{e 1}=300$ effective values to calculate the ANR which accounts for $991 \%$ of energy in transformed domain. This transformation allows the energy to concentrate on the ERDF and achieve a high data compression. This also reduces the amount of computations and storage to describe ANR of scatterers. The antenna for an arbitrary scatterers $\mathbf{r}_{i}^{G C S}$ can then be modeled as

$$
b_{a n t}\left(\mathbf{r}_{i}^{G C S}\right)=\operatorname{vec}\left(\mathbf{R}_{a n t}\right)^{T} \cdot\left(\mathbf{e}_{w}\left(x^{G C S}\right) \otimes \mathbf{e}_{v}\left(y^{G C S}\right) \otimes \mathbf{e}_{u}\left(z^{G C S}\right)\right)
$$

where $\mathbf{e}_{u}(x)=e^{-j x \mathbf{U}}, \mathbf{e}_{v}(y)=e^{-j y \mathbf{V}}, \mathbf{e}_{w}(z)=e^{-j z \mathbf{W}}$ and

$$
\begin{aligned}
& \mathbf{U}=\frac{2 \pi}{M_{x} \delta_{x}} \cdot\left[-\left(M_{x}-1\right) / 2, \ldots,\left(M_{x}-1\right) / 2\right]^{T} \\
& \mathbf{V}=\frac{2 \pi}{M_{y} \delta_{y}} \cdot\left[-\left(M_{y}-1\right) / 2, \ldots,\left(M_{y}-1\right) / 2\right]^{T} \\
& \mathbf{W}=\frac{2 \pi}{M_{z} \delta_{z}} \cdot\left[-\left(M_{z}-1\right) / 2, \ldots,\left(M_{z}-1\right) / 2\right]^{T}
\end{aligned}
$$

Taking advantage of the sparse tensor $\mathbf{R}_{\text {ant }}$, we can calculate the ANR with the nonzero concentration in the ERDF

$$
b_{\text {ant }}\left(\mathbf{r}_{i}^{G C S}\right)=\sum_{m_{e}}^{M_{e}} v_{m_{e}} \cdot a_{s\left(m_{e}, 1\right)}\left(x^{G C S}\right) \cdot b_{s\left(m_{e}, 2\right)}\left(y^{G C S}\right) \cdot c_{s\left(m_{e}, 3\right)}\left(z^{G C S}\right)
$$

where $v_{m_{e}}$ denote the $m_{e}$-th nonzero of ERDF, the subscript $s\left(m_{e}, k\right)$ stands for the $k^{k}$-th subscript of the $m_{e}$-th nonzero in the tensor $\mathbf{R}_{\text {ant }}$ and the operators

$$
a_{s\left(m_{e}, 1\right)}\left(x^{G C S}\right)=e^{-j x u_{s\left(m_{e}, 1\right)}}, b_{s\left(m_{e}, 2\right)}\left(y^{G C S}\right)=e^{-j y v_{s\left(m_{e}, 2\right)}}, c_{s\left(m_{e}, 3\right)}\left(z^{G C S}\right)=e^{-j z w_{s\left(m_{e}, 3\right)}} \text {. }
$$

For notational convenience, we drop the superscript GCS of the coordinates. Using an effective calculation for ANR in the MIMO channel model, (27) yields the improved representation given by 


$$
\mathbf{s}(\boldsymbol{\chi})=\sum_{i=1}^{I} \gamma_{i} \mathbf{a}_{T x}^{U L A}\left(\mathbf{r}_{i}^{A C S 1}\right) \otimes \mathbf{a}_{R x}^{U L A}\left(\mathbf{r}_{i}^{A C S 2}\right) \cdot\left\{\sum_{m_{e}}^{M_{e}} v_{m_{e}} a_{s\left(m_{e}, 1\right)}(x) b_{s\left(m_{e}, 2\right)}(y) c_{s\left(m_{e}, 3\right)}(z)\right\} \otimes \mathbf{a}_{l}\left(\mathbf{r}_{i}\right)
$$

where $\mathbf{a}_{T x}^{U L A}\left(\mathbf{r}_{i}^{A C S 1}\right) \otimes \mathbf{a}_{R x}^{U L A}\left(\mathbf{r}_{i}^{A C S 2}\right)$ describes the influence of antenna array structure. It is separated from the influence of propagation, and model I can alter the antenna array structure conveniently.

3.2.1. Model II: The set of $M_{3 D}$ AAR vectors $\mathbf{b}_{\text {array }}\left(\mathbf{r}_{\left(k_{x}, k_{y}, k_{z}\right)}^{G C S}\right)$ can also be calculated at the spatial sampling grid. The element of the AAR vector $b_{i, j}\left(\mathbf{r}_{\left(k_{x}, k_{y}, k_{z}\right)}^{G C S}\right)$ stands for the ANR of the channel from ${ }^{i}$-th received to ${ }^{j}$-th transmitted antenna. Similarly, the transformed tensor $\mathbf{R}_{i, j}$ of $b_{i, j}\left(\mathbf{r}_{\left(k_{x}, k_{y}, k_{z}\right)}^{G C S}\right)$ can be obtained using (31), and the transformed tensors can be expressed as

$$
\mathbf{R}_{\text {array }}=\left[\begin{array}{c}
\operatorname{vec}\left(\mathbf{R}_{1,1}\right)^{T} \\
\vdots \\
\operatorname{vec}\left(\mathbf{R}_{\mathrm{M}_{R}, \mathrm{M}_{T}}\right)^{T}
\end{array}\right] \in \square^{M_{R} M_{T} \times M_{x} M_{y} M_{z}}
$$

which is referred to as the ERDF of the array.

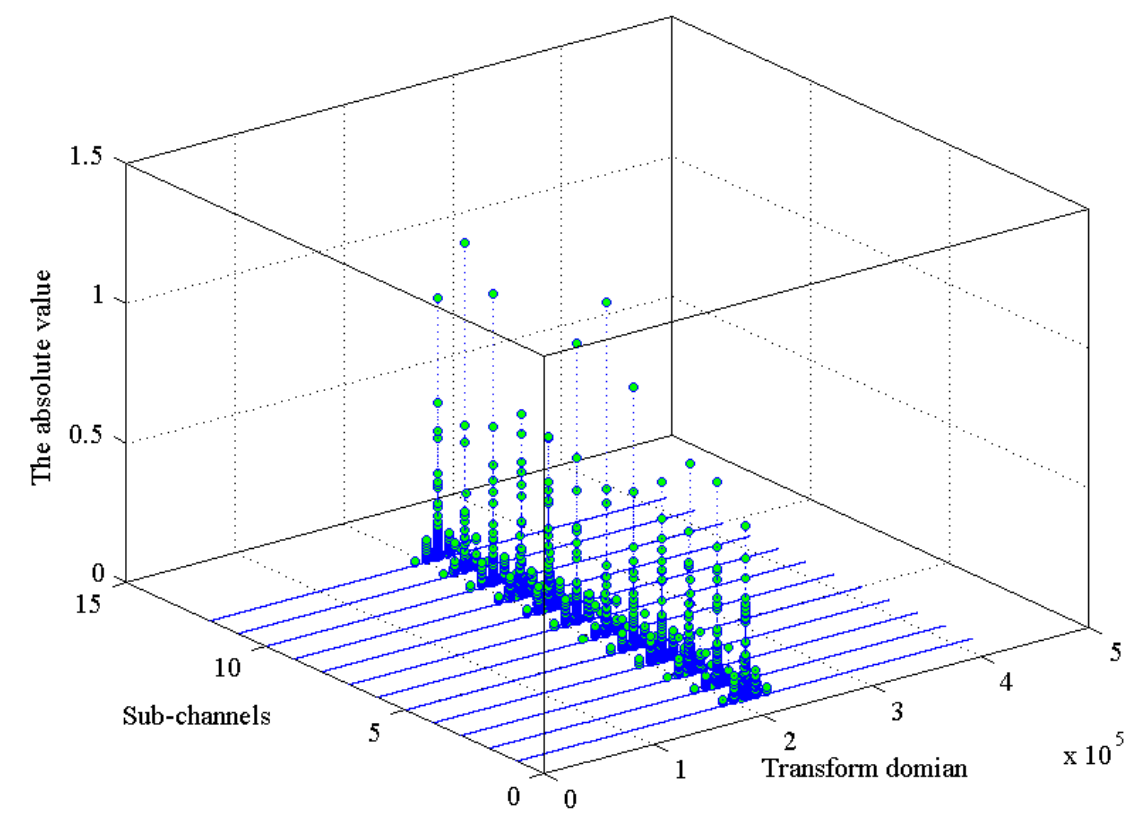

Figure 6. The Absolute Value of AAR-ERDF Expressed by a Matrix which Contains the ANR-ERDF of the Sub-channels Sorted by the Operator Vec() 
Consequently the AAR for an arbitrary scatterers $\mathbf{r}_{i}^{G C S}$ can be expressed

$$
\mathbf{b}_{\text {array }}\left(\mathbf{r}_{i}^{G C S}\right)=\mathbf{R}_{\text {array }} \cdot\left(\mathbf{e}_{w}\left(x^{G C S}\right) \otimes \mathbf{e}_{v}\left(y^{G C S}\right) \otimes \mathbf{e}_{u}\left(z^{G C S}\right)\right)
$$

where $\mathbf{e}_{u}(x)=e^{-j x \mathbf{U}}, \mathbf{e}_{v}(y)=e^{-j y \mathbf{V}}, \mathbf{e}_{w}(z)=e^{-j z \mathbf{W}}$

and

$$
\begin{aligned}
& \mathbf{U}=\frac{2 \pi}{M_{x} \delta_{x}} \cdot\left[-\left(M_{x}-1\right) / 2, \ldots,\left(M_{x}-1\right) / 2\right]^{T} \\
& \mathbf{V}=\frac{2 \pi}{M_{y} \delta_{y}} \cdot\left[-\left(M_{y}-1\right) / 2, \ldots,\left(M_{y}-1\right) / 2\right]^{T} \\
& \mathbf{W}=\frac{2 \pi}{M_{z} \delta_{z}} \cdot\left[-\left(M_{z}-1\right) / 2, \ldots,\left(M_{z}-1\right) / 2\right]^{T}
\end{aligned}
$$

Figure 6 shows the transformed matrix $\mathbf{R}_{\text {array }}$ that contains the energy concentrated ERDF of all ANR of sub-channels and the effective values of AAR-ERDF is approximately $M_{e 2}=13100$. The sparseness of each sub-channel transformed matrix $\mathbf{R}_{i, j}$ result in that the AAR can also be calculated with the nonzero values as

$$
\mathbf{b}_{\text {array }}\left(\mathbf{r}_{i}^{G C S}\right)=\sum_{m_{e}}^{M_{e}} v_{m_{e}} \cdot a_{s\left(m_{e}, 1\right)}\left(x^{G C S}\right) \cdot b_{s\left(m_{e}, 2\right)}\left(y^{G C S}\right) \cdot c_{s\left(m_{e}, 3\right)}\left(z^{G C S}\right)
$$

where $v_{m_{e}}$ is the $m_{e}$-th nonzero value in the sparse matrix $\mathbf{R}_{\text {array }}$ and the subscript

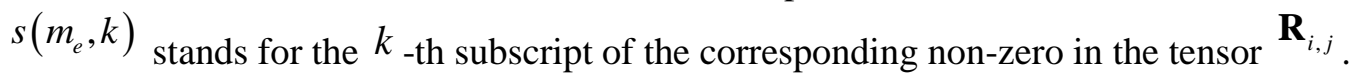

We now substitute the ERDF shown in (37) for array responses in the MIMO channel model (27) and then have

$$
\mathbf{s}(\boldsymbol{\chi})=\sum_{i=1}^{I} \gamma_{i} \cdot\left\{\sum_{m_{e}}^{M_{e}} v_{m_{e}} \cdot a_{s\left(m_{e}, 1\right)}\left(x_{i}\right) \cdot b_{s\left(m_{e}, 2\right)}\left(y_{i}\right) \cdot c_{s\left(m_{e}, 3\right)}\left(z_{i}\right)\right\} \otimes \mathbf{a}_{l}\left(\mathbf{r}_{i}\right)
$$

Since AAR contains the influence of antenna array structure, the proposed model II gives a simple formula to analyze its derivatives with respect to the parameters for some applications, such as gradient based parameter estimation techniques.

\section{Experimented Results}

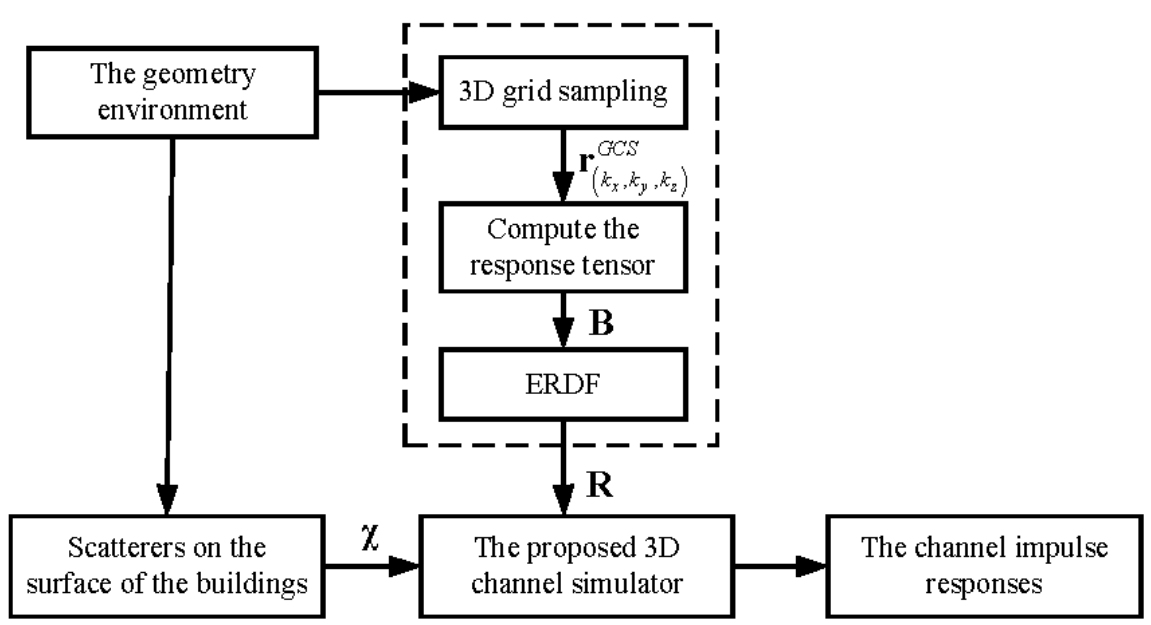

Figure 7. Diagram of the Proposed Model used for 3D Channel Simulation 
The proposed models are used to simulate a 3D MIMO scenario given locations and coefficient of the scatterers (i.e. the set $\chi$ ) shown in Figure 7. A rough position of the scatterers on the surface of the buildings can be achieved as the input parameters of the proposed models according to propagation geometry environment. Another input is the ERDF of the environment. The output is the vector expression of channel impulse response $\mathbf{S}_{\text {Pro }} \in \square^{M_{f} M_{R} M_{T} \times 1}$ shown in (7).

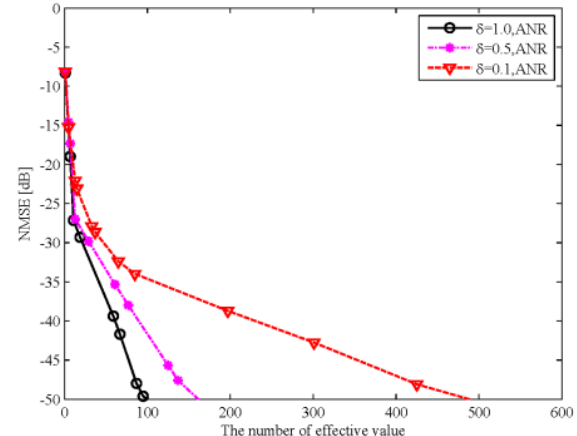

(a)

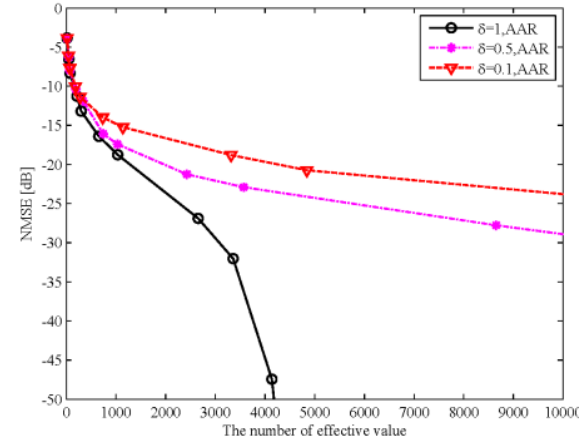

(b)

Figure 8. Normalized Mean Squared Error in dB of (a) the ANR Reconstruction and (b) the AAR Reconstruction for different Sampling Intervals against the Numbers of Effective Values of ERDF

\section{Table 1. The Numbers of Effective Values for ANR and AAR Reconstruction with Different Sampling Intervals $\delta$ for NMSE $=-50 \sim-45[\mathrm{~dB}]$}

\begin{tabular}{|l|l|l|}
\hline & ANR-ERDF & AAR-ERDF \\
\hline$\delta=1$ & $60 \square 100$ & $4000 \square 4200$ \\
\hline$\delta=0.5$ & $140 \square 160$ & $1.2 \times 10^{4} \square 1.4 \times 10^{4}$ \\
\hline$\delta=0.1$ & $400 \square 490$ & $1.6 \times 10^{4} \square 2.0 \times 10^{4}$ \\
\hline
\end{tabular}

As the EADF data of the radiation pattern is stored before use [19], the ERDF can be saved as known data for the scenarios with layout of the coordinate systems (i.e. GCS, ACS1, ACS2) being consistent with the requirement of ERDF. The performance of the proposed model I and model II largely depend on the description of ANR and AAR with the proposed ERDF. Figure 8 shows the Normalized Mean Squared Error (NMSE) for different reconstructions at different sampling intervals and with different numbers of effective values of ERDFs. The ERDFs of the transformed tensor $\mathbf{R}_{\text {ant }}$ and $\mathbf{R}_{\text {array }}$ are calculated differently with $\delta=1, \delta=0.5$ and $\delta=0.1$ as (31) and (35) respectively. For a number of the effective values in $\mathbf{R}_{\text {ant }}$ and $\mathbf{R}_{\text {array }}$, the ANR and AAR are reconstructed as (33) and (37) respectively and are then compared to the originals via the NMSE. The originals of ANR and AAR are obtained using (28) and (29) respectively at all sampling points. Let $\mathbf{B}_{0}$ stands for the originals and $\mathbf{B}_{r}$ be the reconstructed response, then the NMSE is calculated as

$$
N M S E=\frac{E\left[\left|\mathbf{B}_{0}-\mathbf{B}_{r}\right|^{2}\right]}{E\left[\left|\mathbf{B}_{0}\right|^{2}\right]}
$$

Figure 8(a) and Figure 8(b) show how the reconstruction errors of ANR and AAR changes with respect to the numbers of the effective values and sampling intervals 
respectively. Both plots show that a smaller sampling interval need a larger number of effective values at the same NMSE. In other words, a more precise description of the ANR and AAR needs more effective values to reconstruct the details in $3 \mathrm{D}$ space. The number of the effective values for AAR reconstruction is larger than the number of the effective values for ANR reconstruction since the AAR contains the influence of antenna array structure. We focus on the reconstruction errors at $-45 \sim-50[\mathrm{~dB}]$, and some results are illustrated in Table 1. Figure 9 shows the reconstruction errors of ANR and AAR calculated by (39) for different sampling intervals and is plotted for each sampling points in the 3D space. Most scatterer locations in the space keep the low errors and the large errors appear at the edge of the space.

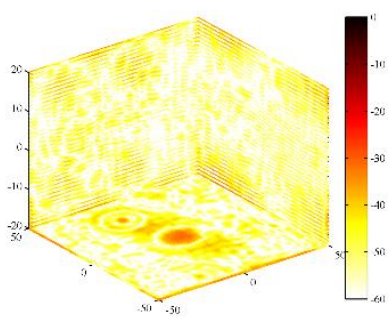

(a)

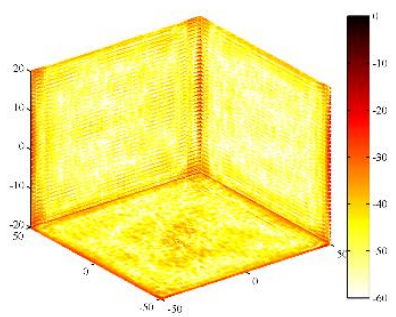

(a)

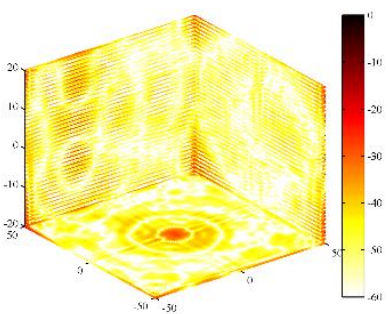

(b)

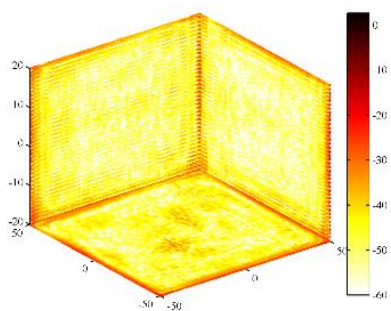

(b)

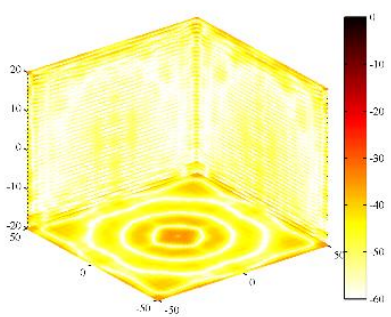

(c)

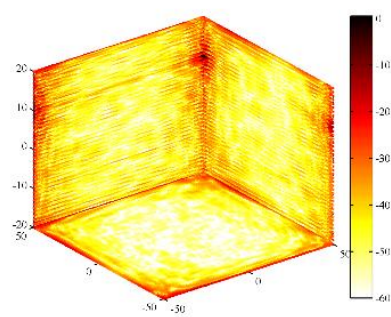

(c)

Figure 9. Reconstruction Error in dB for each Sampling Points in a $100 \times 100$ $\times 40\left[\mathrm{~m}^{3}\right]$ Space with NMSE $=-50 \sim-45$ [dB]. Plots (a), (b), (c) for an ANR Reconstruction Done with $\delta=1, \delta=0.5$ and $\delta=0.1$ Respectively and (d), (e) (f) for a Sub-channel of AAR Reconstruction Done with $\delta=1, \delta=0.5$ and $\delta=0.1$ respectively

Table 2. Parameter for Channel Simulation

\begin{tabular}{|c|c|c|c|}
\hline Number of scatterers & $60 \sim 72$ & $\mathbf{r}_{T}$ & $(30,-10,1)$ \\
\hline Center frequency & $5.2 \mathrm{GHz}$ & $\mathbf{r}_{R}$ & $(0,0,4)$ \\
\hline Wideband & $200 \mathrm{MHz}$ & $\mathbf{M}_{\mathbf{f}}$ & 256 \\
\hline Time & $25 \mathrm{~s}$ & $\mathbf{S N R}$ & $20 \mathrm{~dB}$ \\
\hline Scenarios & \multicolumn{3}{|c|}{$100 \times 100 \times 40\left[\mathrm{~m}^{3}\right]$} \\
\hline Antennas & $\begin{array}{l}\text { ULA at Tx and Rx side, with 3 elements and 4 } \\
\text { elements respectively as vertical dipole }\end{array}$ \\
\hline
\end{tabular}

In order to evaluate the performance of the proposed models using ERDF, the channel simulation is based on a geometry-based MIMO channel simulator IlmProp which mimics most characteristics of typical outdoor real measured channels [29, 30]. By user interaction of IlmProp, the positions and motions of the scatterers can be set. The simulator outputs the channel impulse responses of the corresponding synthetic scenario. 
Note that the main principle of the simulator is based on the geometry-based channel model (6) [28], which we refer to as the reference model.

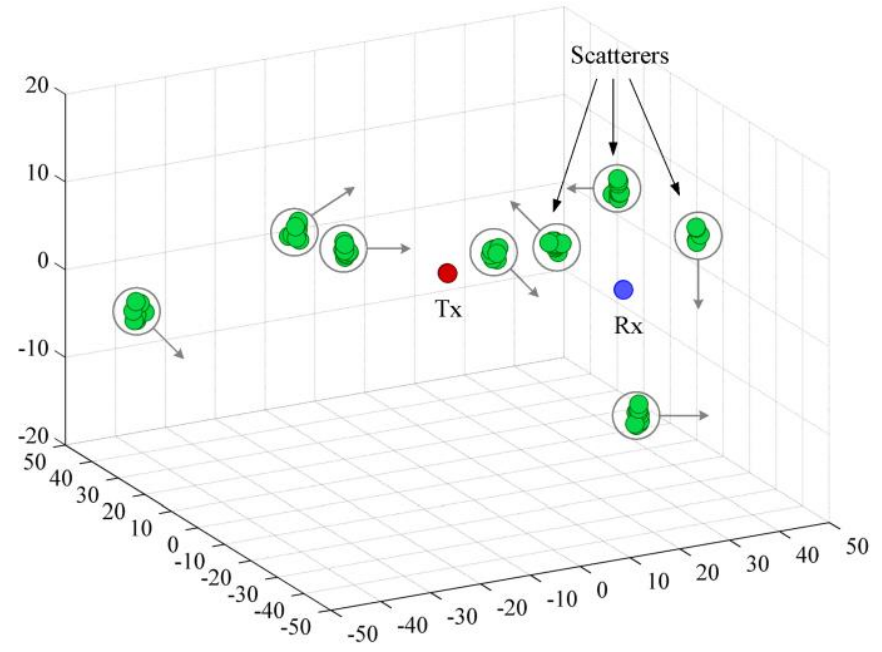

Figure 10. Simple Dynamic Scenario in a $100 \times 100 \times 40\left[\mathrm{~m}^{3}\right]$ Space with the Positions of the 72 Scatterers Changing in Time
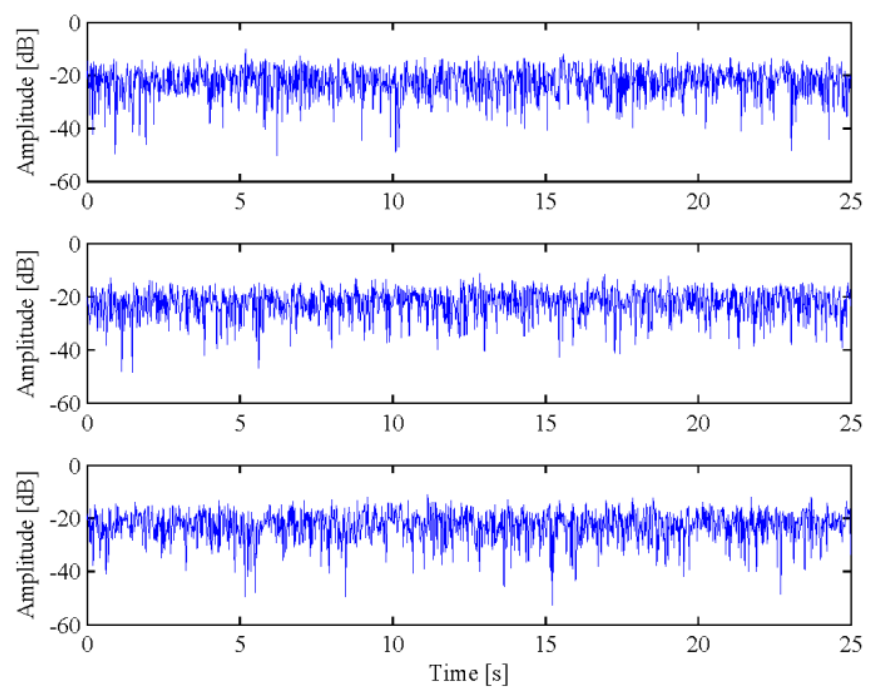

Figure 11. Amplitudes of the CIRs for a Specific Frequency Bin Plotted in dB Against Time for the Reference Model (top), Model I (middle) and Model II (bottom)

In Figure 10, a simple scenario with 72 moving scatterers and two fixed Tx and Rx in a 3D space $100 \times 100 \times 40\left[\mathrm{~m}^{3}\right]$ is given. The scatterers are moving in time along the direction represented by arrowed line. The locations and coefficients of all 72 scatterers (i.e. the set $\mathcal{\chi}$ ) are random but known as the input parameters for proposed models and the simulator Ilmprop. The other input parameters are given in Table 2, except the number of scatterers is 72 here. Then the outputs channel impulse responses (CIRs) generated by the proposed models and the Ilmprop can be compared. Figure 11 shows the CIRs for a specific frequency $f_{0}$ plotted against time for $25 \mathrm{~s}$. The fast-fading characteristic of CIR 
generated by the proposed model I and model II are consistent with the CIR from the simulator Ilmprop.

In order to assess the models quantitatively, we compute the mutual information (MI) as a metric for the models. The MI of wideband channel is obtained by averaging over the frequency domain as [31]

$$
I(\mathrm{t})=\frac{1}{B} \sum_{m=1}^{M_{f}} \log _{2} \operatorname{det}\left(\mathbf{I}_{M_{R}}+\frac{S N R}{M_{T}} \mathbf{H}_{n}\left(t, f_{m}\right) \mathbf{H}_{n}^{H}\left(t, f_{m}\right)\right)
$$

where the sum is calculated over the frequency bins $f_{m}=m \cdot f_{0}+f_{c} . \mathbf{I}_{M_{R}}$ denotes the $M_{R} \times M_{R}$ identity matrix, and $\mathbf{H}_{n}\left(t, f_{m}\right)$ the normalized $M_{R} \times M_{T}$ MIMO channel matrix. The normalization is done such that every time instant is normalized separately as

$$
\mathbf{H}_{n}\left(t, f_{m}\right)=\frac{1}{\frac{1}{M_{f}} \sum_{m=0}^{M_{f}}\left\|\mathbf{H}\left(t, f_{m}\right)\right\|_{F}^{2}} \mathbf{H}\left(t, f_{m}\right)
$$

Here, the metric reflects the spatial structure of the channel.

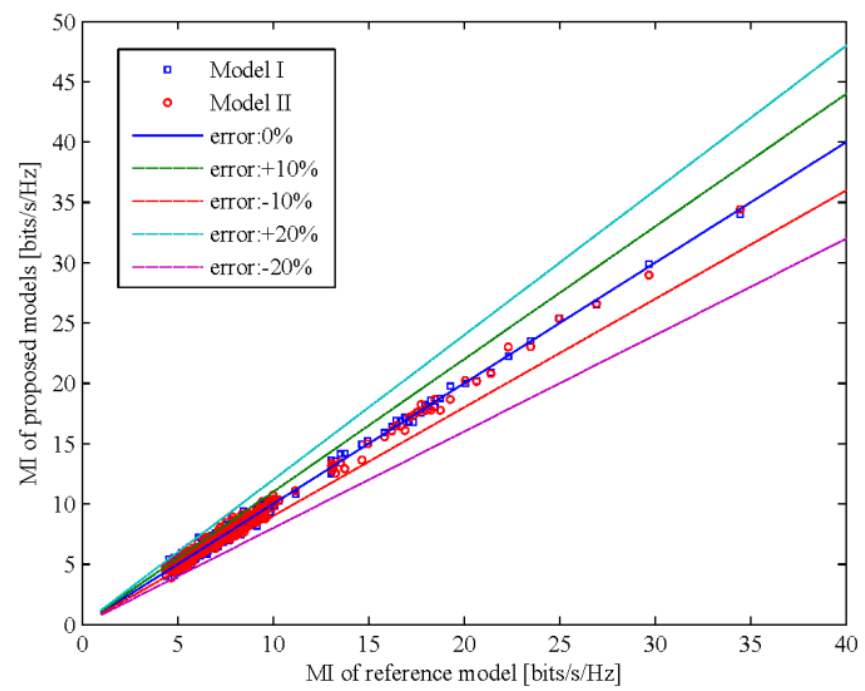

Figure 12. Mutual Information of the Reference vs. the Proposed Models for 256 different Scenarios at a Receive SNR of $20 \mathrm{~dB}$

A number of synthetic scenarios are generated by IlmProp with the setup summarized in Table 2. The proposed models are used to simulate the scenarios with the same setup. Let $\mathbf{H}^{\text {Ilm }}$ stands for the output of IlmProp, $\mathbf{H}_{1}^{\text {Pro }}$ and $\mathbf{H}_{2}^{\text {Pro }}$ be the output of the proposed models. The MIs of the models are calculated as (40). Figure 12 shows the results of the comparison: scatter plots of the MI of the reference model versus the proposed model for MIMO are depicted. For each model, a specific marker corresponds to one of the 256 scenarios. We see that the MI of Model I and Model II are consistent with the MI of the Reference model from the simulator. Thus the proposed models and the conventional geometry-based model have equivalent performance in the simulation results. 


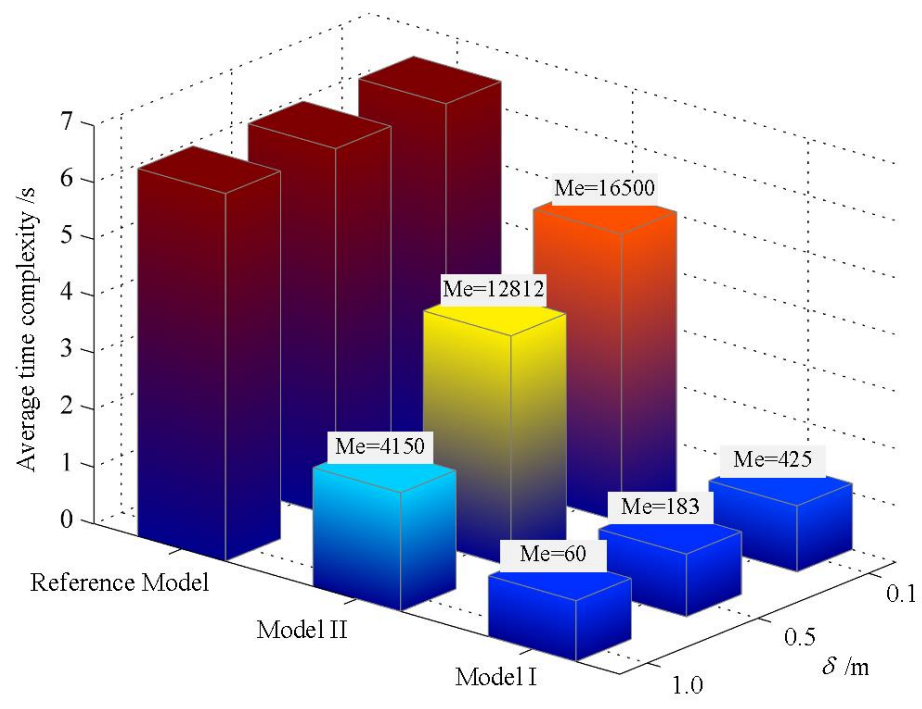

\section{Figure 13. The Simulation Time Comparison between the Reference Model and the Proposed Models in Different Sampling Intervals}

Figure 13 compare the computation time of the proposed model (34) and (38) with the reference model (6) for simulating the scenario shown in Figure 10. The scenario parameters are the same as given in Table 2. The EADF of the reference model and the ERDF of the proposed model require a re-sampling of the beam pattern and preprocessing. Here both of them is stored as the known data after the pre-processing calculations. The EADF are stored in a $L_{1} \times L_{2}$ matrix for the reference model (6), where $L_{1}=L_{2}=27$ [19]. According to Table 1, the number of effective values of ERDF marked at the top of the bar ensures a small reconstruction error. Three sampling intervals of the proposed models are investigated. Using Matlab with Core i3 CPU $3 \mathrm{GHz}$ and $4 \mathrm{~GB}$ RAM, we find that the simulation time is different between the proposed models due to the different quantities of effective values. However, the computational time of the proposed models are lower than that of the reference model. Because the reference model need to transform the scatterers locations to the delay and angular information of a path, whereas the proposed models can use the scatterers locations to generate the CIRs directly.

\section{Summary and Conclusion}

This paper chooses the scatterers as parameters to character the 3D MIMO channel. It is based on the propagation mechanisms and establishes a physical model, taking the spatial distribution of scatterers and the antenna array structure effects into consideration. The proposed model I is used for separating the influence of the antenna arrays from the propagation channel whereas the model II is simpler and more convenient to analyze its derivatives with respect to the parameters for some applications.

The proposed models allows us to generate channels with all possible scatteres' distribution on the surface of buildings. As geographical features are stored in the REM database, ergodic channel simulation method becomes advantageous to comprehend the channel, such as, a ergodic positioning of the scatterers allows us to find out which propagation conditions own a desired frequency selectiveness as well as other specific traits of channel. 


\section{References}

[1] H. Celebi, I. Guvenc, S. Gezici and H. Arslan, "Cognitive-radio systems for spectrum, location, and environmental awareness", IEEE Antennas and Propagation Magazine, vol. 52, no. 4, (2010), pp. 41-61.

[2] B. Wang and K. R. Liu, "Advances in cognitive radio networks: A survey." IEEE Journal of Selected Topics in Signal Processing, vol. 5, no. 1, (2011), pp.5-23.

[3] Y. P. Zhao, "Enabling Cognitive Radios through Radio Environment Maps", USA: Doctor Dissertation of Virginia Polytechnic Institute and State University, (2007).

[4] A. F. Bruce, "Cognitive Radio Technology", Elsevier Publisher, (2009).

[5] K. M. Richard and T. Ryan, "Algorithms and Bounds for Estimating Location, Directionality, and Environmental Parameters of Primary Spectrum Users", IEEE Transactions on Wireless Communications, vol. 8, no. 11, (2009), pp. 5692- 5701.

[6] H. B. Yilmaz, T. Tugcu and S. Bayhan, "Radio environment map as enabler for practical cognitive radio networks", Communications Magazine, IEEE, vol. 51, no. 12, (2013), pp. 162-169.

[7] A. F. Molisch, L. J. Greenstein and M. Shafi, "Propagation issues for cognitive radio", "Proceedings of the IEEE", vol. 97, no. 5, (2009), pp. 787-804.

[8] H. Asplund, A. A. Glazunov, A. F. Molisch, K. Pedersen and M. Steinbauer, "The COST 259 directional channel model-part II: macrocells", Wireless Communications, IEEE Transactions on, vol. 5, no. 12, (2006), pp. 3434-3450.

[9] G. D. Durgin, V. Kukshya and T. S. Rappaport, "Wideband measurements of angle and delay dispersion for outdoor and indoor peer-to-peer radio channels at $1920 \mathrm{MHz}$ ", Antennas and Propagation, IEEE Transactions on, vol. 51, no. 5, (2003), pp.936-944.

[10] P. Almers, E. Bonek, A. Burr, N. Czink, M. Debbah and D. Esposti, "Survey of channel and radio propagation models for wireless MIMO systems", EURASIP Journal on Wireless Communications and Networking, vol. 2007, no. 1, (2007), pp.56-56.

[11] Z. Parizi, M. Mehrjoo and A. Shokouh, "A Survey of Geometrically-Based MIMO Propagation Channel Models", (2013).

[12] J. Poutanen, F. Tufvesson, K. Haneda, V. M. Kolmonen and P. Vainikainen, "Multi-link MIMO channel modeling using geometry-based approach", Antennas and Propagation, IEEE Transactions on, vol. 60, no. 2, (2012), pp.587-596.

[13] M. Steinbauer, A. F. Molisch and E. Bonek, "The double-directional radio channel", Antennas and Propagation Magazine, IEEE, vol. 43, no. 4, (2001), pp. 51-63.

[14] R. Bhagavatula, C. Oestges and R. W. Heath, "A new double-directional channel model including antenna patterns, array orientation, and depolarization", Vehicular Technology, IEEE Transactions on, vol. 59, no. 5, (2010), pp.2219-2231.

[15] K. Kalliola, H. Laitinen, P. Vainikainen, M. Toeltsch, J. Laurila and E. Bonek, E, "3-D doubledirectional radio channel characterization for urban macrocellular applications", Antennas and Propagation, IEEE Transactions on, vol. 51, no. 11, (2003), pp. 3122-3133.

[16] A. F. Molisch, "A generic model for MIMO wireless propagation channels in macro-and microcells", Signal Processing, IEEE Transactions on, vol. 52, no. 1, (2004), pp. 61-71.

[17] A. F. Molisch, "Ultrawideband propagation channels-theory, measurement, and modeling", Vehicular Technology, IEEE Transactions on, vol. 54, no. 5, (2005), pp.1528-1545.

[18] A. Richter, "Estimation of radio channel parameters: Models and algorithms", ISLE, (2005).

[19] M. Landmann and G. Del Galdo, "Efficient antenna description for MIMO channel modelling and estimation", European Microwave Week, Amsterdam, The Netherlands, (2004).

[20] M. Costa, V. Koivunen and A. Richter, "Low complexity azimuth and elevation estimation for arbitrary array configurations", Acoustics, Speech and Signal Processing, 2009. ICASSP 2009. IEEE International Conference on, (2009), pp. 2185-2188.

[21] F. Belloni, A. Richtera and V. Koivunen, "DoA estimation via manifold separation for arbitrary array structures", Signal Processing, IEEE Transactions on, vol. 55, no. 10, (2007), pp. 4800-4810.

[22] M. Costa, A. Richter and V. Koivunen, "Unified array manifold decomposition based on spherical harmonics and 2-D Fourier basis", Signal Processing, IEEE Transactions on, vol. 58, no. 9, (2010), pp. 4634-4645.

[23] Y. Zhao, S. Mao, J. O. Neel and J. H. Reed, "Performance evaluation of cognitive radios: Metrics, utility functions, and methodology", Proceedings of the IEEE, vol. 97, no. 4, (2009), pp. 642-659.

[24] A. Kuchar, J. P. Rossi and E. Bonek, "Directional macro-cell channel characterization from urban measurements", Antennas and Propagation, IEEE Transactions on, vol. 48, no. 2, (2000), pp. 137-146.

[25] J. Zhang, C. Pan, F. Pei, G. Liu and X. Cheng, "Three-dimensional fading channel models: A survey of elevation angle research", Communications Magazine, IEEE, vol. 52, no. 6, (2004), pp. 218-226.

[26] A. Kammoun, H. Khanfir, Z. Altman, M. Debbah and M. Kamoun, "Survey on 3D channel modeling: From theory to standardization", arXiv preprint, (2013).

[27] X. H. Wu, A. A. Kishka and A. W. Glisson, "Antenna effects on a monostatic MIMO radar for direction estimation, a Cramer-Rao lower bound analysis", Antennas and Propagation, IEEE Transactions on, vol. 59, no. 6, (2011), pp. 2388-2395. 
[28] G. Del Galdo, "Geometry Based Channel Modeling for Multi-user MIMO Systems and Applications", ISLE, (2007).

[29] http://www2.tu-ilmenau.de/nt/en/ilmprop//

[30] G. Del Galdo and M. Haardt, "IlmProp: A flexible geometry-based simulation environment for multiuser MIMO communications", COST 273 Proj. Rep. TD (03), (2003), p. 188.

[31] N. Czink, "The random-cluster model: a stochastic MIMO channel model for broadband wireless communication systems of the 3rd generation and beyond", (2007).

\section{Authors}

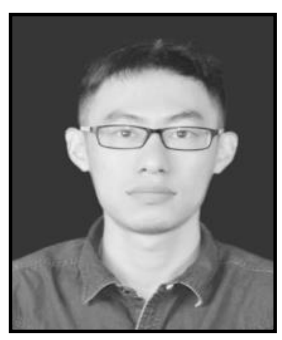

Sheng Deng, he received the B.S. degree from Nanchang University, Nanchang, China in 2013 and received the M.S. degree from Nanchang University, Nanchang, China in 2016. His research interests include radio measurement and channel modeling, complex environment sensing, processing, and wireless communications.

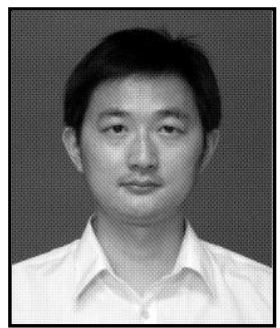

Wang Yuhao, he received the Ph.D. degree in space physics from Wuhan University, Wuhan, China, in 2006. He is currently a Professor with Cognition Sensor Network Laboratory, School of Information Engineering, Nanchang University, Nanchang, China. His research interests include radio measurement and channel modeling, software-defined radio, complex environment sensing and monitoring, nonlinear signal processing, multimedia, sensor networks, and wireless communications.

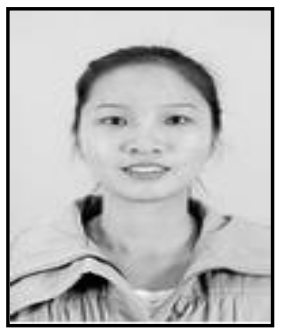

Yu jiangnan received the B.S. degree from Nanchang University, Nanchang, China in 2012 and received the M.S. degree from Nanchang University, Nanchang, China in 2015. Her research interests include radio measurement and channel modeling, complex environment sensing, processing, and wireless communications.

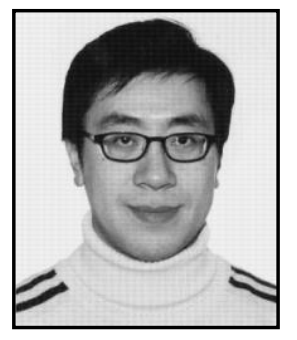

Henry Leung, he received the Ph.D. degree in electrical and computer engineering from McMaster University, Hamilton, ON, Canada. He is currently a Professor with the Department of Electrical and Computer Engineering, University of Calgary, Calgary, AB, Canada. His research interests include chaos, computational intelligence, data mining, information fusion, nonlinear signal processing, multimedia, sensor networks, and wireless communications.

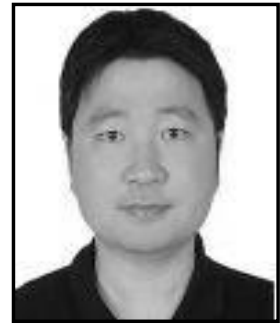

Huilin Zhou, he received the Ph.D. degree from Wuhan University, Wuhan, China. He is currently a Professor with the Department of Information Engineering, Nanchang University, Nanchang, China. His research interests include radar imaging, radar signal processing and target identification. 


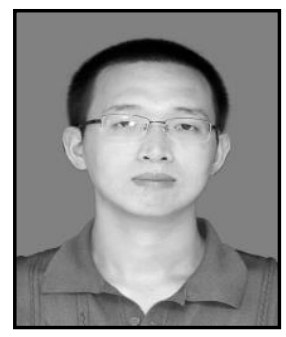

Bo Kong, he received the Ph.D. degree in space physics from Nanchang University, Nanchang, China. His research interests include wireless communications, compressed sensing and passive radar. 
International Journal of Future Generation Communication and Networking Vol. 9, No.10, (2016) 